\title{
1 Bacterial profiles and their antimicrobial susceptibility pattern of 2 Isolates from inanimate hospital environments at Tikur Anbessa 3 Specialized Teaching Hospital, Addis Ababa, Ethiopia

5 Shemse Sebre', 2 *, Woldaregay Erku1, Aminu Seman1, 2, Tewachw Awoke ${ }^{2,}$ 3, Zelalem 
27

28

29

30

\section{Abstract}

Microbial contamination of hospital environment plays an important role in the spread of health care-associated infections (HCAIs). This study was conducted to determine bacterial contamination, bacterial profiles and antimicrobial susceptibility pattern of bacterial isolates from environmental surfaces and medical equipment. A cross-sectional study was conducted at Tikur Anbessa Specialized Hospital (TASH) from June to September, 2018. A total of 164 inanimate surfaces located at intensive care units (ICUs) and operation theaters (OTs) were swabbed. All isolates were identified by using routine bacterial culture, Gram staining and a panel of biochemical tests. For each identified bacteria, antibiogram profiles were determined by the Kirby Bauer disk diffusion method according to the guidelines of the Clinical and Laboratory Standards Institute (CLSI). Out of the 164 swabbed samples, 141 (86\%) were positive for bacterial growth. The predominant bacteria identified from OTs and ICUs were S. aureus (23\% vs $11.5 \%)$, Acinetobacter spp (3.8\% vs $17.5 \%$ ) and Coagulase negative Staphylococcus (CONS) (12.6\% vs 2.7\%) respectively. Linens were the most contaminated materials among items studied at the hospital (14.8\%). The proportions of resistance among Gram-positive bacteria (GPB) were high for penicillin $(92.8 \%)$, cefoxitin $(83.5 \%)$ and erythromycin $(54.1 \%)$. However, the most effective antibiotics were clindamycin with only $10.4 \%$ and $16.5 \%$ resistance rates, respectively. The antimicrobial susceptibility profiles of Gram-negative bacteria (GNB) revealed that the most effective antibiotics were amikacin, ciprofloxacin, and gentamicin with resistance rate of $25 \%$, $37.5 \%$, and $46.3 \%$, respectively. However, the highest resistance was recorded against ampicillin (97.5\%), ceftazidime (91.3\%), ceftriaxone (91.3\%) and aztreonam (90\%). The inanimate surfaces near immediate patient environment and commonly touched medical equipment within OTs and ICUs are reservoirs of potential pathogenic bacteria that could predispose critically ill patients to acquire HCAIs. The proportions of antimicrobial resistance profile of the isolates are much higher from studied clean inanimate environments.

Keywords: Antimicrobial susceptibility pattern, Operation theaters, Inanimate Hospital environments, Intensive care unit, Bacteria. 


\section{Introduction}

58 Hospital environment represents a new ecological place for medically important nosocomial pathogens, antibiotic-resistant microorganisms and reservoirs of resistance gene, which have been commonly, found on various surfaces within hospitals (e.g. medical equipment, housekeeping surfaces, workplaces and lobby (furniture) [1,2]. Studies investigating hospital environments reported that pathogens were ubiquitous in all hospital units but the interest was usually focused on intensive care and operation unit, especially due to the vulnerability of patients in these units [3]. There is also high antibiotic usage and invasive procedure from these units [1].

Bacterial cross-contamination plays an important role in health care-associated infections (HCAIs) and resistant strain dissemination [1, 4]. The majority of the HCAIs are believed to be transmitted directly from patient to patient, but increasing evidence demonstrates that also the medical personnel as well as the clinical environment (i.e., surfaces and equipment) often are a source of infections [5]. Hospital design and hygienic practices have been largely directed at controlling nosocomial pathogens and resistant strains contaminating air, hands, equipment, and surfaces [6]. A better understanding of how bacterial cross-contamination occurs can provide the basis for the development of evidence-based preventive measures [4].

74 Emergence of multi-drug resistant (MDR) strains in a hospital environment; particularly in 75 developing countries, is an increasing problem which is an obstacle for management of HCAIs

76 [7-10]. In Ethiopia, studies reported high prevalence of HCAIs mainly due to MDR pathogens 77 including the country's largest tertiary referral Hospitals [11-13], which warrants the critical need for a reassessment of the role played by inanimate environment in the transmission of 79 nosocomial infections [6, 14].

80 Studies on the bacterial contaminations of ward of the hospital environments in Ethiopia reported

81 high bacterial load and multidrug resistant (MDR) strains [9, 10, 15, 16]. However, few data 82 exist on the bacterial contamination of the hospital environment in the studied hospital. 83 Therefore, the aim of this study were to determine bacterial contamination, detect potential 
pathogenic bacteria and to determine the antimicrobial susceptibility patterns from inanimate hospital environments in the environments of Operation Theaters (OTs) and Intensive Care Units (ICUs) at Tikur Anbessa Specialized Teaching Hospital in Addis Ababa, Ethiopia.

\section{Materials and Methods}

\section{Study setting, Study period and Sampling locations}

A cross-sectional study was conducted at Tikur Anbessa Specialized Hospital (TASH), Addis Ababa, Ethiopia from June to September, 2018. TASH is a tertiary hospital and major referral center for other hospitals in Ethiopia. TASH has 800 beds and provides care for approximately 370,000-400,000 patients per year. The samples were collected from four intensive care units including Surgical, Pediatric, Medical and Medical-Surgical units. A total of seven operating theaters were examined including Emergency, Neurology, Endo-Renal, Obstetrics and gynaecology, Pediatrics, Cardio-Vascular and Gastro intestinal tract (GIT) units.

\section{Surfaces sampling}

The detection of bacteria in ICUs and OTs were performed by using the swab method from surfaces and medical devices. All samples were collected every morning after cleaning of the hospital environment was completed. Moreover, samples in OTs were collected before start of operations. Sampling sites around a bed in each ICUs and OTs were chosen based on the frequency with which the surfaces were touched. Sterile swabs were moistened in Brain Heart Infusion (BHI) and then, were used to swab (i) commonly touched medical equipment including beds, monitors, OR-light, linens, ventilators, oxygen supply, anesthesia machine, suction buttons and Laparoscopy (ii) workstation, including keyboards, computer mice; (iii) environments including floors, wall and corridors; (iv) Lobby (furniture) including chair, table, lockers and trowels; (v) Sinks; (vi) hospital textiles including bed linen based on methods described previously [17-20].

\section{Microbiology Analysis}

Each swab sample was pre-enriched in sterile $\mathrm{BHI}$ and incubated at $37^{\circ} \mathrm{C}$ for 24 hours. A loop full of the turbid broth was then sub-cultured on blood agar (Oxoid, UK), Mannitol salt agar (MSA), MacConkey agar and Chromagar TM Strep B base plates (Chromagar microbiology, 
112 France). Differential and selective characteristics for each agar medium were recorded for the 113 initial screening of suspected potential pathogens. Furthermore, specific colony color (mauve 114 color) on Chromagar TM Strep B was considered for Group B Streptococci (GBS) while yellow 115 colony color on MSA was considered for S. aureus.

116 Gram-negative bacteria were further identified by Gram stain and standard biochemical tests like 117 Triple Sugar Iron Agar (TSI), urea, citrate, Sulfide Indole Motility (SIM) medium, growth in 118 Lysine Iron Agar (LIA), Mannitol, malonate, and oxidase test. On the other hand, Gram-positive 119 bacteria were further identified by Gram stain, optochin, bacitracin, CAMP test and different 120 biochemical tests such as catalase, coagulase, bile esculin and salt tolerance test described based on hand book of Clinical Microbiology Procedures [21].

\section{Antimicrobial susceptibility testing}

123 Antimicrobial susceptibility testing of the isolates were performed using 21 antibiotics (Oxoid, UK) based on the Kirby-Bauer disk diffusion method on Mueller-Hinton agar (MHA) (Oxoid, UK) and Mueller-Hinton with blood agar (Oxoid, UK) for Streptococci spp and Enterococcus spp [22]. An inoculum for each isolate was prepared by emulsifying colonies from an overnight pure culture in sterile normal saline $(0.85 \%)$ in test tubes with the turbidity adjusted to 0.5 McFarland standards. The bacterial suspension was uniformly streaked on MHA plates using sterile swabs and left for 3 minutes prior to introduction of the antibiotics.

For Gram-negative bacteria the following antibiotics were used (in $\mu \mathrm{g} / \mathrm{disk}$ ): ampicillin (10), amoxicillin and clavulanic acid (10/10), ceftriaxone (30), cefotaxime (30), ceftazidime (30), amikacin (30), gentamicin (10), ciprofloxacin (5), sulfamethoxazole-trimethoprim (1.25/23.75), cefoxitin (30), cefuroxime (30), cefepime (30), piperacillin-tazobactam (100/10), meropenem (10) and aztreonam (30) based on Clinical Laboratory Standards Institute (CLSI) [22].

On the other hand, for Gram-positive bacteria antibiotics (in $\mu \mathrm{g} / \mathrm{disk}$ ) selected for susceptibility testing included penicillin (10 units), gentamicin (10), erythromycin (15), ciprofloxacin (5), doxycycline (30), vancomycin (30), cefoxitin (30), sulfamethoxazole-trimethoprim (1.25/23.75), clindamycin (2) and chloramphenicol (30). The plates were incubated at $35{ }^{\circ} \mathrm{C}$ for $24 \mathrm{~h}$, and the diameters of zone of inhibition were measured with Vernier caliper and results were reported as susceptible (S), intermediate (I), or resistant (R), according to CLSI guidelines [22]. 


\section{Quality Assurance}

142 To ensure the quality of the result from different assays, internal quality assurance systems was

143 in place for all laboratory procedures and double checking of the result was done. All the 144 methods to be used were validated as fit for the purpose before use in the study. Standard 145 operating procedures (SOPs) were used for specific purpose for all laboratory procedures. 146 Quality control strains of Enterococcus faecalis ATCC $^{\circledR} 29212$, S. aureus ATCC ${ }^{\circledR} 25923$, E. coli $147 \mathrm{ATCC}^{\circledR}$ 2592, K. pneumoniae $\mathrm{ATCC}^{\circledR} 1705$ and $K$. pneumoniae $\mathrm{ATCC}^{\circledR} 1706$ were used to 148 confirm the result of antibiotics, media and to assess the quality of the general laboratory 149 procedure [22].

\section{Statistical analysis}

151 Data analysis was performed using Stata version 14 software program (Stata Corporation, 152 Lakeway Drive, College Station, Texas), and descriptive statistics (percentages or frequency) 153 was calculated. A difference was considered statistically significant for P-value $\leq 0.05$.

\section{Ethics approval}

155 The study protocol was approved by the Department of Microbiology, Immunology and 156 Parasitology Research Ethics Review Committee (DRERC), College of Health Sciences, Addis 157 Ababa University (Ref. no. DRERC/17/18/02-G). Prior to sample collection, written approval 158 was obtained from administrative unit of Tikur Anbessa Specialized Hospital. 
166

167

168

169

170

171

172 bed and linens.

\section{Results}

\section{Culture Results}

During the four months study, a total of 164 environmental swabs were collected in the studied OTs ( $\mathrm{n}=99)$ and ICUs $(\mathrm{n}=65)$ of the hospital. Of these swab samples, $141(86 \%)$ were positive for bacterial growth, from which a total of 183 bacterial isolates were identified. Multi-bacterial contamination was detected in $26.8 \%$ of the samples, mainly found on the surfaces of ventilators,

\section{Frequency of bacterial etiologies}

174 Out of the 183 bacterial isolates, 103(56.3\%) were Gram-positive bacteria (GPB) and the rest 175 Gram-negative bacteria (GNB). Among the GPB S. aureus (34.4\%), CONS (15.3\%) and Bacillus 176 spp (3.3\%) were the dominant isolates. Among the GNB Acinetobacter spp (21.3\%), 177 Pseudomonas spp (7.7\%) and E. coli (4.9\%) were the dominant isolates. Overall, S. aureus was 178 the most frequently isolated bacteria (34.4\%) followed by Acinetobacter spp (21.3\%) and CONS 179 (15.3\%) (Table 1).

Table 1: The frequency of isolated bacteria at TASH, 2018

\begin{tabular}{ll}
\hline Isolates & $\mathbf{N}(\mathbf{\%})$ \\
\hline Gram-negative & $\mathbf{8 0 ( 4 3 . 7 )}$ \\
Acinetobacter spp & $39(21.3)$ \\
Pseudomonas spp & $14(7.7)$ \\
E. coli & $9(4.9)$ \\
Serratia spp & $4(2.2)$ \\
Klebsiella pneumoniae & $6(3.3)$ \\
Klebsiella oxytoca & $4(2.2)$ \\
Others* & $4(2.2)$ \\
Gram-positive & $\mathbf{1 0 3}(\mathbf{5 6 . 3})$ \\
S. aureus & $63(34.4)$ \\
CONS & $28(15.3)$ \\
Bacillus spp & $6(3.3)$ \\
Streptococcus agalactiae & $3(1.6)$ \\
Enterococcus spp & $3(1.6)$ \\
\hline
\end{tabular}




\section{Distribution of bacterial isolates between ICUs and OTs}

184 Most of the potential bacterial pathogens were isolated from Intensive care units (ICUs), 50.3\%

185 (92/183). Significant differences between Gram-positive and Gram-negative bacteria were 186 observed between wards in OTs (39.9\% vs 9.8\%) and ICUs (16.4\% vs 33.9\%) respectively $187(p=0.000)$. The ICUs were mainly contaminated with GNB, 67.4\% (62/92), of which the 188 predominant ones being Acinetobacter spp accounting for 34.8\% (32/92) followed by $S$. aureus 189 with $22.8 \%(21 / 92)$ isolation rate. Most of the bacteria in ICUs were isolated from Medical190 Surgical $(16.4 \%, 30 / 183)$ ward. The major pathogens in this ICU were S. aureus from GPB and 191 Acinetobacter spp from GNB, each with isolation rate of $(33.3 \%, 10 / 16)$. The Operation Theaters 192 (OTs) were mainly contaminated by GPB, 80.2\% (73/91). The major pathogens in the theatre 193 were S. aureus, 46.2\% (42/91) and CONS, 25.3\% (23/91). Endo-Renal theatre was mostly 194 contaminated with $S$. aureus with rate as high as $31.3 \%(5 / 16)$ (Table 2$)$. 
Table 2: Distribution of potential pathogenic bacteria between ICUs and OTs at TASH, 2018

\begin{tabular}{|c|c|c|c|c|c|c|c|c|c|c|c|}
\hline \multirow[b]{2}{*}{ Bacteria } & \multicolumn{4}{|c|}{ ICUs $(\mathrm{N}=92)$} & \multicolumn{7}{|c|}{ OTs $(\mathrm{N}=91)$} \\
\hline & $\begin{array}{l}\text { Surgical } \\
\text { n (\%) }\end{array}$ & $\begin{array}{l}\text { Pediatric } \\
\text { n (\%) }\end{array}$ & $\begin{array}{l}\text { Medical } \\
\text { n (\%) }\end{array}$ & $\begin{array}{l}\text { Medical- } \\
\text { Surgical } \\
\text { n (\%) }\end{array}$ & $\begin{array}{l}\text { Emergency } \\
\text { n (\%) }\end{array}$ & $\begin{array}{l}\text { Neurology } \\
\text { n (\%) }\end{array}$ & $\begin{array}{l}\text { Endo- } \\
\text { Renal } \\
\text { n (\%) }\end{array}$ & $\begin{array}{l}\text { Gyn-obs } \\
\text { n (\%) }\end{array}$ & $\begin{array}{l}\text { Pediatric } \\
\text { n (\%) }\end{array}$ & $\begin{array}{l}\text { Cardo-Vs } \\
\text { n (\%) }\end{array}$ & $\begin{array}{l}\text { GIT } \\
\text { n (\%) }\end{array}$ \\
\hline S. aureus & $4(25)$ & $4(16.7)$ & $3(13.6)$ & $10(33.3)$ & $8(66.7)$ & $7(53.8)$ & $5(31.3)$ & $6(60)$ & $4(33.3)$ & $6(42.9)$ & $6(42.9)$ \\
\hline CONS & $1(6.3)$ & $0(0)$ & $3(13.6)$ & $1(3.3)$ & $4(33.3)$ & $2(15.4)$ & $0(0)$ & $3(30)$ & $6(50)$ & $6(42.9)$ & $2(14.3)$ \\
\hline Bacillus spp & $0(0)$ & $0(0)$ & $0(0)$ & $0(0)$ & $0(0)$ & $2(15.4)$ & $0(0)$ & $1(10)$ & $0(0)$ & $0(0)$ & $3(21.4)$ \\
\hline Enterococcus spp & $0(0)$ & $1(4.2)$ & $1(4.5)$ & $0(0)$ & $0(0)$ & $0(0)$ & $0(0)$ & $0(0)$ & $1(8.3)$ & $0(0)$ & $0(0)$ \\
\hline GBS & $0(0)$ & $0(0)$ & $0(0)$ & $2(6.7)$ & $0(0)$ & $1(7.7)$ & $0(0)$ & $0(0)$ & $0(0)$ & $0(0)$ & $0(0)$ \\
\hline Acinetobacter spp & $6(37.5)$ & $6(25)$ & $10(45.5)$ & $10(33.3)$ & $0(0)$ & $0(0)$ & $4(25)$ & $0(0)$ & $1(8.3)$ & $1(7.1)$ & $1(7.1)$ \\
\hline Pseudomonas spp & $2(12.5)$ & $5(20.8)$ & $2(9.1)$ & $0(0)$ & $0(0)$ & $1(7.7)$ & $2(12.5)$ & $0(0)$ & $0(0)$ & $0(0)$ & $2(14.3)$ \\
\hline Klebsiella spp & $1(6.3)$ & $4(16.7)$ & $2(9.1)$ & $2(6.7)$ & $0(0)$ & $0(0)$ & $2(12.5)$ & $0(0)$ & $0(0)$ & $0(0)$ & $0(0)$ \\
\hline E. coli & $1(6.3)$ & $4(16.7)$ & $1(14.5)$ & $3(10)$ & $0(0)$ & $0(0)$ & $0(0)$ & $0(0)$ & $0(0)$ & $0(0)$ & $0(0)$ \\
\hline Serratia spp & $1(6.3)$ & $0(0)$ & $0(0)$ & $0(0)$ & $0(0)$ & $0(0)$ & $2(12.5)$ & $0(0)$ & $0(0)$ & $1(7.1)$ & $0(0)$ \\
\hline Others * & $0(0)$ & $0(0)$ & $0(0)$ & $2(6.7)$ & $0(0)$ & $0(0)$ & $1(6.3)$ & $0(0)$ & $0(0)$ & $0(0)$ & $0(0)$ \\
\hline Total, N (\%) & $16(8.7)$ & $24(13.1)$ & $22(12)$ & $30(16.4)$ & $12(6.6)$ & $13(7.1)$ & $16(8.7)$ & $10(5.5)$ & $12(6.6)$ & $14(7.7)$ & $14(7.7)$ \\
\hline
\end{tabular}


205 The highest bacterial contaminated samples were taken from bed linens followed by 206 environmental surfaces and bed. Linens were mostly contaminated with Klebsiella spp., (54.5\%, 207 6/27), followed by Acinetobacter spp., (15.4\%, 6/39). Beds were mainly contaminated with 208 S. aureus $(12.7 \%, 8 / 63)$. Sinks were mainly colonized by $S$. aureus $(7.7 \%, 6 / 63)$, Pseudomonas $209 \operatorname{spp}(7.1 \%, 1 / 14)$ and Acinetobacter spp (5.1\%, 2/39). Klebsiella spp is mainly contaminated 210 ventilators $(27.3 \%, 3 / 11)$ (Table 3$)$. 
Table 3: Distribution of bacteria over different surfaces in ICU and OTs at TASH, Addis Ababa, Ethiopia, 2018

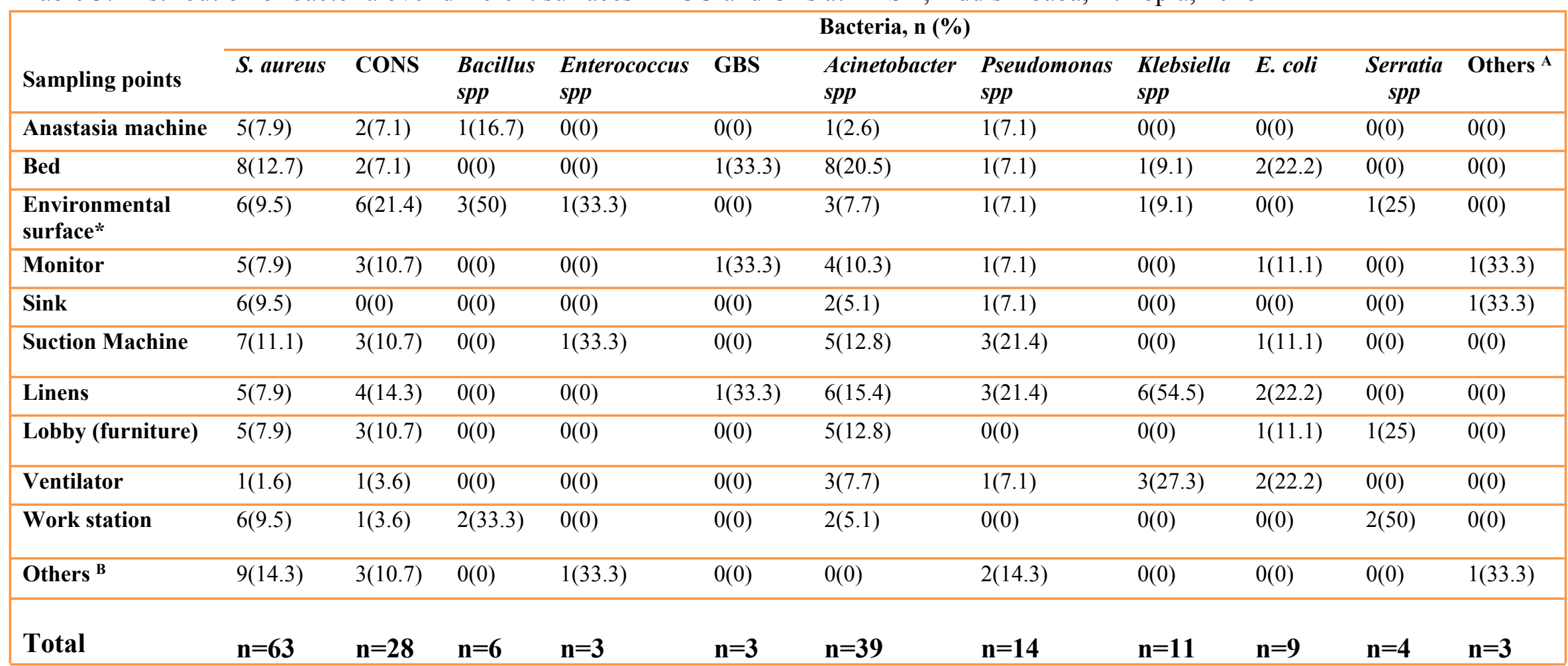

Others ${ }^{\mathrm{A}}$ (Shigella spp, Enterobacter spp); Others ${ }^{\mathrm{B}}$ (Laparoscopy, OR-Light, oxygen cylinder, Trowels); *Environmental surfaces (Door knob, Floor, Corroder and Wall), Group B Streptococcus (Streptococcus agalactiae), CONS: Coagulase negative staphylococci. 
215 The proportions of antimicrobial resistance among GPB were high for penicillin (92.8\%), 216 cefoxitin (83.5\%) and erythromycin (54.1\%). Low level of resistance was recorded for 217 clindamycin (10.4\%) and gentamicin (16.5\%). Using cefoxitin disk as a surrogate marker, 218 54(85.7\%) of Staphylococcus aureus isolates were defined as MRSA. High resistance level was 219 also recorded to penicillin (93.7\%). Vancomycin resistance was demonstrated by 12 (19\%) $S$. 220 aureus, 5 (17.9\%) CONS and 1(33.3\%) Enterococcus spp (Table 4).

221

222

223 
Table 4: Antimicrobial susceptibility pattern of Gram-positive bacteria at TASH, 2018.

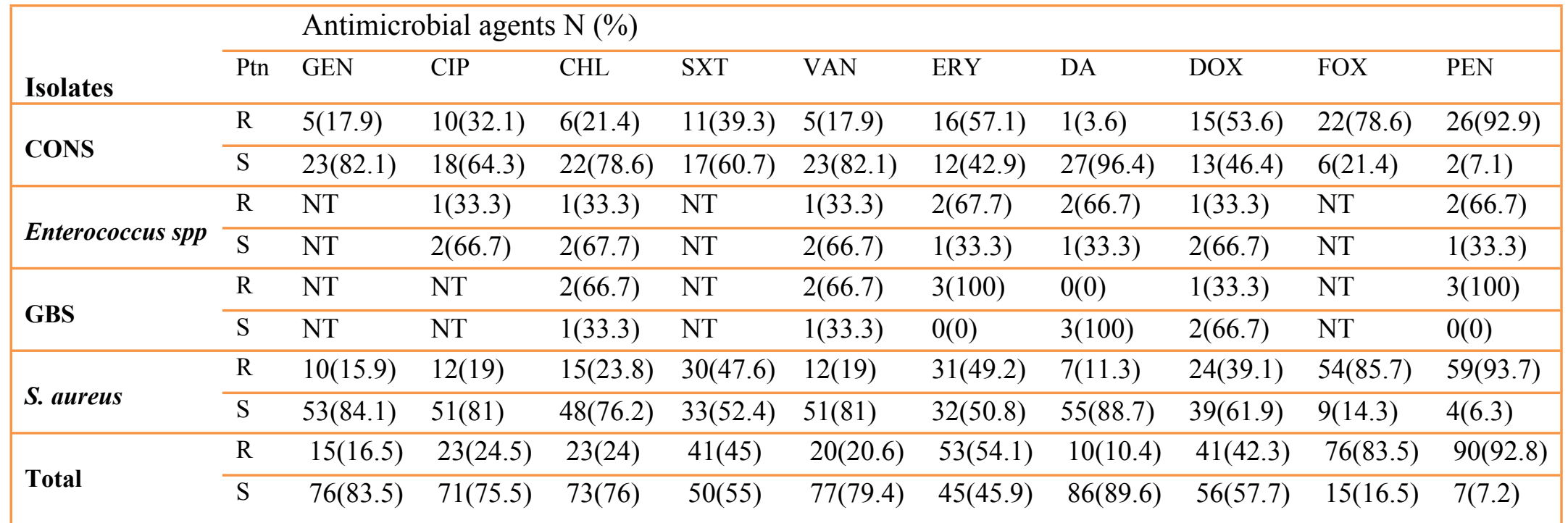

N: Number of tested strains; R: Resistant; S: Sensitive; Ptn: Pattern; FOX: Cefoxitin; GEN: Gentamicin; CIP: Ciprofloxacin; CHL: Chloramphenicol; SXT: Trimethoprim-Sulfamethoxazole; VAN: Vancomycin; ERY: Erythromycin; DA: Clindamycin; DOX: Doxycycline; PEN: Penicillin, NT: Not tested, GBS : Group B Streptococcus (Streptococcus agalactiae), CoNS: Coagulase negative staphylococci 


\section{Antibiogram profile for Gram-negative isolates}

236 Most of the GNB exhibited significantly high resistance to most of the tested antibiotics; for 237 example, ampicillin (97.5\%), ceftazidime (91.3\%), ceftriaxone (91.3\%) and aztreonam (90\%), 238 cefotaxime (83.8\%), amoxicillin and clavulanic acid (77.6\%) and cefoxitin (76.3\%). Similarly, 239 significant resistance level was also recorded for cefepime (75\%), sulfamethoxazole240 trimethoprim (71.3\%), piperacillin-tazobactam (68.7\%) and meropenem (56.3\%). Low level 241 resistance was recorded for amikacin (25\%), ciprofloxacin (37.5\%) and gentamicin (46.3\%).

242 Acinetobacter spp showed the highest resistance level to almost all tested antibiotics including 243 penicillin, cephalosporins, and carbapenems and monobactam groups of antibiotics including: 244 ampicillin (100\%), aztreonam (100\%), ceftazidime (100\%), amoxicillin and clavulanic acid 245 (100\%), ceftriaxone (97.4\%) and cefotaxime (92.3\%). Low resistance level by Acinetobacter spp 246 was recorded to amikacin (25\%) (Table 5). 
Table 5: Antimicrobial susceptibility pattern of Gram-negative bacteria at TASH, 2018

\begin{tabular}{|c|c|c|c|c|c|c|c|c|c|c|c|c|c|c|c|}
\hline & & \multicolumn{14}{|c|}{ Antimicrobial agent's n (\%) } \\
\hline Isolates & Ptn & AMP & AZM & CTX & CRO & CTZ & FOX & FEP & AMC & TZP & MRP & $\mathrm{AK}$ & GEN & CIP & SXT \\
\hline \multirow{2}{*}{ Acinetobacter spp } & $\mathrm{R}$ & $39(100)$ & $39(100)$ & $36(92.3)$ & $38(97.4)$ & $39(100)$ & $37(94.8)$ & $34(87.2)$ & $32(100)$ & $34(87.2)$ & $29(74.4)$ & $14(25)$ & $27(69.2)$ & $18(46.1)$ & $31(79.5)$ \\
\hline & $\mathrm{S}$ & $0(0)$ & $0(0)$ & $3(7.7)$ & $1(2.6)$ & $0(0)$ & $2(5.2)$ & $5(12.8)$ & $5(0)$ & $5(12.8)$ & $10(25.6)$ & $25(75)$ & $12(30.8)$ & 21(53.9) & $8(20.5)$ \\
\hline \multirow{2}{*}{ E. coli } & $\mathrm{R}$ & $9(100)$ & $6(66.7)$ & $7(77.8)$ & $7(77.8)$ & $7(77.8)$ & $4(44.4)$ & $7(77.7)$ & $5(55.4)$ & $5(55.6)$ & $3(33.3)$ & $2(22.2)$ & $4(44.4)$ & $4(44.4)$ & $7(77.8)$ \\
\hline & $\mathrm{S}$ & $0(0)$ & $3(33.3)$ & $2(22.2)$ & $2(22.2)$ & $2(22.2)$ & $5(55.6)$ & $2(33.3)$ & $4(44.6)$ & $4(44.4)$ & $6(66.7)$ & $7(77.8)$ & $5(55.6)$ & $5(55.6)$ & $2(22.2)$ \\
\hline \multirow{2}{*}{ Enterobacter spp } & $\mathrm{R}$ & $2(100)$ & $2(100)$ & $2(100)$ & $2(100)$ & $2(100)$ & $2(100)$ & $2(100)$ & $2(100)$ & $2(100)$ & $1(50)$ & $0(0)$ & $1(50)$ & $2(100)$ & $2(100)$ \\
\hline & $\mathrm{S}$ & $0(0)$ & $0(0)$ & $0(0)$ & $0(0)$ & $0(0)$ & $0(0)$ & $0(0)$ & $0(0)$ & $0(0)$ & $1(50)$ & $2(100)$ & $1(50)$ & $0(0)$ & $0(0)$ \\
\hline \multirow{2}{*}{ K. oxytoca } & $\mathrm{R}$ & $4(100)$ & $3(75)$ & $3(75)$ & $4(100)$ & $3(75)$ & $3(75)$ & $3(75)$ & $1(50)$ & $2(50)$ & $1(25)$ & $1(25)$ & $1(25)$ & $2(50)$ & $2(50)$ \\
\hline & S & $0(0)$ & $1(25)$ & $1(25)$ & $0(0)$ & $1(25)$ & $1(25)$ & $1(25)$ & $1(50)$ & $2(50)$ & $3(75)$ & $3(75)$ & $3(75)$ & $2(50)$ & $2(50)$ \\
\hline \multirow{2}{*}{ K. pneumoniae } & $\mathrm{R}$ & $5(83.3)$ & $4(66.7)$ & $4(66.7)$ & $6(100)$ & $5(83.7)$ & $3(50)$ & $5(83.3)$ & $6(100)$ & $4(66.7)$ & $4(66.7)$ & $3(50)$ & $2(33.3)$ & $2(33.4)$ & $5(83.3)$ \\
\hline & $\mathrm{S}$ & $1(16.7)$ & $2(33.3)$ & $2(33.3)$ & $0(0)$ & $1(16.3)$ & $3(50)$ & $1(16.7)$ & $0(0)$ & $2(33.3)$ & $2(33.3)$ & $3(50)$ & $4(66.7)$ & $4(66.6)$ & $1(16.7)$ \\
\hline \multirow{2}{*}{ K. rhino } & $\mathrm{R}$ & $1(100)$ & $1(100)$ & $1(100)$ & $1(100)$ & $1(100)$ & $0(0)$ & $1(100)$ & $1(100)$ & $0(0)$ & $0(0)$ & $0(0)$ & $0(0)$ & $0(0)$ & $0(0)$ \\
\hline & S & $0(0)$ & $0(0)$ & $0(0)$ & $0(0)$ & $0(0)$ & $1(100)$ & $0(0)$ & $0(0)$ & $1(100)$ & $1(100)$ & $1(100)$ & $1(100)$ & $1(100)$ & $1(100)$ \\
\hline \multirow{2}{*}{ Pseudomonas spp } & $\mathrm{R}$ & $13(92.9)$ & $13(92.9)$ & $12(85.7)$ & $12(85.7)$ & $11(78.6)$ & $11(78.5)$ & $5(35.7)$ & $8(57.1)$ & $5(35.7)$ & $6(42.8)$ & $0(0)$ & $2(14.3)$ & $2(14.3)$ & $8(57.2)$ \\
\hline & $\mathrm{S}$ & $1(7.1)$ & $1(7.1)$ & $2(14.3)$ & $2(14.3)$ & $3(21.4)$ & $3(21.5)$ & $9(64.3)$ & $6(42.9)$ & $9(64.3)$ & $8(57.2)$ & $14(100)$ & $12(85.7)$ & $12(85.7)$ & $6(43.7)$ \\
\hline \multirow{2}{*}{ Serratia spp } & $\mathrm{R}$ & $4(100)$ & $3(75)$ & $1(25)$ & $2(50)$ & $4(100)$ & $1(25)$ & $3(75)$ & $3(75)$ & $2(50)$ & $1(25)$ & $0(0)$ & $0(0)$ & $0(0)$ & $1(25)$ \\
\hline & $\mathrm{S}$ & $0(0)$ & $1(25)$ & $3(75)$ & $2(50)$ & $0(0)$ & $3(75)$ & $1(25)$ & $1(25)$ & $2(50)$ & $3(75)$ & $4(100)$ & $4(100)$ & $4(100)$ & $3(75)$ \\
\hline \multirow{2}{*}{ Shigella spp } & $\mathrm{R}$ & $1(100)$ & $1(100)$ & $1(100)$ & $1(100)$ & $1(100)$ & $0(0)$ & $0(0)$ & $1(100)$ & $1(100)$ & $0(0)$ & $0(0)$ & $0(0)$ & $0(0)$ & $1(100)$ \\
\hline & $\mathrm{S}$ & $0(0)$ & $0(0)$ & $0(0)$ & $0(0)$ & $0(0)$ & $1(100)$ & $1(100)$ & $0(0)$ & $0(0)$ & $1(100)$ & $1(100)$ & $1(100)$ & $1(100)$ & $0(0)$ \\
\hline \multirow{2}{*}{ Total } & $\mathrm{R}$ & $78(97.5)$ & $72(90)$ & $67(83.8)$ & 73(91.3) & 73(91.3) & $61(76.3)$ & $60(75)$ & $59(77.6)$ & $55(68.7)$ & $45(56.3)$ & $20(25)$ & $37(46.3)$ & $30(37.5)$ & $57(71.3)$ \\
\hline & $\mathrm{S}$ & $2(2.5)$ & $2(10)$ & $13(16.2)$ & $7(8.7)$ & $7(8.7)$ & $19(23.7)$ & $20(25)$ & $17(22.4)$ & $25(31.3)$ & $35(43.7)$ & $60(75)$ & $43(53.7)$ & $50(62.5)$ & $23(28.7)$ \\
\hline
\end{tabular}

N: Number of tested strains; R: resistant; S: Sensitive; \%: percentage; Ptn: Pattern; AMP: ampicillin; AZT: Aztreonam; CTX : cefotaxime; CRO: ceftriaxone; CTZ: ceftazidime; FOX: Cefoxitin; FEP: Cefepime; AMC: Amoxicillin and clavulanic acid ; CHL: Chloramphenicol; MRP: Meropenem; AK: Amikacin; GEN: Gentamicin; CIP: ciprofloxacin; SXT: Sulfamethoxazole + trimethoprim 


\section{Discussions}

In the present study, out of 164 fomites and medical devices samples from swabs of normally clean hospital environments, $141(86 \%)$ were positive for bacterial contamination. Our result agreed with other reports where bacterial contamination was found to be very high such as report from Zimbabwe (86.2\%) [14] and from Morocco (96.3\%) [23]. In contrast to our result, lower bacterial contaminations were observed from studies conducted elsewhere; Gaza Strip (24.7\%) [24], Sudan (29.7\%) [25], Uganda (44.2\%) [26], Nigeria (39.4\%) [27] and Bahir Dar, Northwest Ethiopia (39.6\%) [16]. Differences in hand hygiene, ventilation system, sterilisation and disinfection techniques could account for these discrepancies [1, 28, 29].

Higher levels of bacterial contamination observed in our study could be attributed primarily to the use of ineffective disinfectants during surface cleaning, and inadequate uses of standard precautions such as hand hygiene and contact precautions, as well as migration of the organisms through air flow or other means particularly in places where the ventlation system has not been not in place or not working properly [20]. Infrequent cleaning of inanimate surfaces and medical equipments could also contribute to poor microbial quality of the hospital surfaces [14, 30, 31]. This situation is prominently linked to hospitals which show unwillingness to put funds into contamination control such as the ventilation systems, those that lack information about the level of contamination and ineffectiveness of commonly used disinfectants in their hospital, and those with inappropriate waste controls.

The results of our study showed substantial contamination of hospital inanimate environments by varied groups of bacteria, including both Gram-positive (56.3\%) and Gram-negative (43.7\%). Comparable to our results, frequency of GPB from other studies in Ethiopia and abroad proved to be constituted the leading contaminating bacteria compared to GNB; for example, in Gondar, Ethiopia (60.5\% vs 39.5\%) [32], in Northwest, Ethiopia (81.6\% vs 18.4\%) [16], in Iran (60.7\% vs $39.3 \%)$ [33] and in Nigeria (52.2\% vs 47.8\%) [34]. The dominance of GPB could be explained by the fact that these bacteria, being devoid of lipid-dominant desiccation prone outer membrane, have natural ability to retain their viability on abiotic hospital environments for several days to months [29, 33]. 
However, in contrast to our results, several authors from different countries reported that GNB were isolated more frequently than Gram-positive ones: for example, Zimbabwe (66.2\% vs 33.82\%) [14], Gaza Strip (51.6\% vs 48.4\%) [24] and Morocco (73.3\% vs 26.7\%) [23]. These variations may be due to different sampling times (e.g. during endemic vs outbreak situations), the presence of already colonized and/or infected patients during sampling, the use of different sampling techniques and culture methodologies, and variation in specific hospital sampling sites (e.g., OTs vs ICUs) [35-38]. In fact, in agreement to the latter reasoning, more GNB (67.4\%; 62/92) than Gram-positive ones were obtained from ICUs inanimate environment even our 297 finding.

298 Overall, S. aureus was the most frequently isolated bacteria (39.8\%) followed by Acinetobacter spp (18.9\%) and CONS (15.5\%). S. aureus and CONS were also the most frequently isolated bacteria from previous other studies such as Ethiopia [39], Nigeria [34] and Zaria, Nigeria [27]. S. aureus constitute part of the normal human flora, inhabiting the skin, mucous membranes [40] and regularly shed onto the hospital environment by patients and medical personnel, whereupon they persist [14]. This isolates were also considered as the potential pathogenic bacteria that result in nosocomial infections and indicators of inadequate clinical surface hygiene in hospital environments [17, 25, 41]. Moreover, these bacteria were also resistant to common disinfectant methods and hence spread easily in the environment, which enables them to colonize and infect the patients receiving health care service at the facility [24, 33].

Among the different hospital environments and hospital items examined, the highest bacterial contaminated samples were taken from bed linens, environmental surface and beds, similar to the observations from other studies in Ethiopia and abroad [3, 27, 33, 36]. Bed linens and bed were 312 mainly contaminated by Acinetobacter spp (20.5\% and 15.4\%), CONS (7.1\% and 14.3\%), and S. 313 aureus (12.7\% and 7.9\%), respectively. Comparable results were obtained on beds and linens 314 samples from studies conducted in Iran [33] and Nigeria [27]. The sources of such 315 contaminations could be cross-contamination from a patient's flora, health care workers' hands, 316 contaminated storage carts, or due to contamination during the washing process especially that of bed linens [33, 35, 37]. 
In our study, sinks were mainly colonized by $S$. aureus $(7.7 \%, 6 / 63)$, Pseudomonas spp (7.1\%, 1/14) and Acinetobacter spp (5.1\%, 2/39), which is in line with several reports that hospital associated outbreaks in critical care wards occur largely due to the opportunistic pathogen [14, 27, 42]. This could be linked to the fact that the moist hospital environments, particularly sinks, are conducive for persistence of these bacteria, which are known to have the ability to form biofilms in water, sinks, toilets, showers and drains [43, 44]. Moreover, acquisition of multiple virulence determinants and intrinsic resistance to commonly used antibiotics and disinfectants by these pathogens may result in maintaining their viability and hence persistence under such harsh environments $[43,45]$.

Bloodstream infection and ventilator-associated pneumonia especially in the intensive care units are usually linked to device contamination such as central venous catheters, urinary catheters and ventilators [46]. In our study, ventilators were frequently contaminated by Klebsiella spp $(27.3 \%, 3 / 11)$, which was also reported from a study conducted in Iran (54.4\%, 6/11) [33]. Source of contamination of ventilators by $K$. pneumoniae might be from the aspiration of secretions from the oropharynx of colonized patients, where staff hands may act as the transmission vehicle $[47,48]$.

In regards to antimicrobial resistance profile of the isolates, our results showed high proportions of drug resistance, where most of the GNB were highly resistant to most of the tested antibiotics such as ampicillin (97.5\%), ceftazidime (91.3\%), ceftriaxone (91.3\%), aztreonam (90\%), cefotaxime (83.8\%), cefoxitin (76.3\%), and amoxicillin and clavulanic acid (77.6\%), which is in line with similar resistance rates from other studies conducted elsewhere like Gaza in Palestine [24], Morocco [3] and Sudan [25]. Increased resistance to $\beta$-lactams antibiotics is due to the selective pressure exerted by the antibiotics [49]. Because these tested antimicrobials represent the antibiotics most frequently used in practice, serious problems can be encountered while prescribing those antibiotics [3]. One way of fighting such a rise of resistance should include establishing guidelines for prescribing antibiotics [16] based on locally generated antimicrobial resistance data such as the findings from this study.

On the other hand, low resistance level was recorded to non-beta-lactam antimicrobials such as, amikacin (25\%) and ciprofloxacin (37.5\%). Comparable results were recorded from studies conducted from Sudan for amikacin (23.5\%) and ciprofloxacin (42.7\%) [25]. Still lower 
resistance rate was documented for these two antibiotics in Palestine for amikacin (6.1\%) and ciprofloxacin (27.3\%) [24], possibly an area where they may not routinely be prescribed for community and/or hospital acquired infections.

Not surprisingly, GPB demonstrated elevated resistance to penicillin $(92.8 \%)$, cefoxitin $(83.5 \%)$ and erythromycin (54.1\%). Similarly, high resistance level was also reported from Ethiopia by a Meta- analysis study for penicillin and erythromycin with a pooled resistance level of $99.1 \%$ and $97.2 \%$, respectively [50]. Moreover, similar resistance level was also reported from Uganda for penicillin (93\%) [26]. Of the 64 S. aureus isolates obtained in this study, 54 (85.7\%) were MRSA, which is close to the rate reported from Zimbabwe (100\%) [14], although much higher than the rate from Uganda (52\%) [51].

In this study, vancomycin resistance was demonstrated by 12 (19\%) S. aureus (VRSA), 5(17.9\%) CONS and 1(33.3\%) Enterococcus spp. Vancomycin resistant Staphylococci were also reported in a study from Zimbabwe, where $40 \%$ of $S$. aureus and $23.5 \%$ of CONS were vancomycin resistant, despite its scarcity in usage [14]. It has been suggested that patients at risk for VRSA are co-infected or co-colonized with VRE and MRSA, which enables conjugative transfer of vanA gene from VRE to MRSA in a biofilm environment leading to a VRSA strain $[33,52]$.

\section{Conclusions}

In this study, bacterial samples were sought for and isolated only from the environmental surfaces; not from patients and hands of health professionals. S. aureus, Acinetobacter spp and CONS form the majority of the environmental contaminants most likely to cause HAIs. We concluded that special attention to infection control policies, antimicrobial resistance screening, good clinical practice and cleaning techniques are needed to reduce the potential risk of pathogenic bacteria and resistant strain transmission among hospital staff and patients. Our results may be indicative evidence that bacterial environmental contamination is possibly contributing to HAIs and MDR strain dissemination in the hospital environment and further large scale investigations are needed. 


\section{$376 \quad$ Additional files}

377 S 1 Table: Morphological and biochemical characterization of gram-positive bacteria isolated from

378 environmental samples at Tikur Anbessa Specialized Hospital, Ethiopia, 2018 (DOC 35 kb).

379 S 2 Table: Morphological and biochemical characterization of gram-negative bacteria isolated from

380 environmental samples at Tikur Anbessa Specialized Hospital, Ethiopia, 2018 (DOC 35 kb).

381 S 3 Table: Data description (DOC $17 \mathrm{~kb}$ ).

$382 \quad$ Funding

383 This research work were financed by Addis Ababa University and Armauer Hansen research 384 institute, Addis Ababa, Ethiopia. The funder had no role in study design, data collection and 385 analysis, decision to publish, or preparation of the manuscript.

\section{Acknowledgements}

387 The authors here by thank Addis Ababa University (AAU) and Armauer Hansen Research 388 Institute (AHRI) for their financial and material support and Tikur Anbessa Specialized Hospital 389 staff.

\section{Competing interests}

391 The authors declare that they have no competing interests.

\section{Data Availability}

393 The dataset supporting the findings of this article have been attached as supplementary 394 information files. 


\section{Reference}

1. Mora M, Mahnert A, Koskinen K, Pausan MR, Oberauner-Wappis L, Krause R, et al. Microorganisms in confined habitats: microbial monitoring and control of intensive care units, operating rooms, cleanrooms and the International Space Station. Frontiers in microbiology. 2016;7:1573.

2. Rozman U, Šostar Turk S. PCR Technique for the Microbial Analysis of Inanimate Hospital Environment. 2016. doi: 10.5772/65742.

3. Bakkali M, Hmid K, Kari K, Zouhdi M, Mzibri M. Characterization of Bacterial Strains and their Resistance Status in Hospital Environment. J Trop Dis. 2015;4(180):2.

4. Loftus RW, Brown JR, Koff MD, Reddy S, Heard SO, Patel HM, et al. Multiple reservoirs contribute to intraoperative bacterial transmission. Anesthesia \& Analgesia. 2012;114(6):1236-48.

5. Morales L, Rodriguez C, Gamboa-Coronado MDM. Molecular detection of Clostridium difficile on inert surfaces from a Costa Rican hospital during and after an outbreak. Am J Infect Control. 2016;44(12):1517-9. doi: 10.1016/j.ajic.2016.09.003. PubMed PMID: 28340959.

6. Otter JA, Yezli S, French GL. The role played by contaminated surfaces in the transmission of nosocomial pathogens. Infect Control Hosp Epidemiol. 2011;32(7):687-99. doi: 10.1086/660363. PubMed PMID: 21666400.

7. Gebremariam TT, Declaro MFF. Operating theaters as a source of nosocomial infection: a systematic review. Saudi Journal for Health Sciences. 2014;3(1):5.

8. Tesfaye T, Berhe Y, Gebreselassie K. Microbial contamination of operating Theatre at Ayder Referral Hospital, Northern Ethiopia. International Journal of Pharma Sciences and Research (IJPSR). 2015;6(10).

9. Solomon FB, Wadilo FW, Arota AA, Abraham YL. Antibiotic resistant airborne bacteria and their multidrug resistance pattern at University teaching referral Hospital in South Ethiopia. Annals of clinical microbiology and antimicrobials. 2017;16(1):29.

10. Engda T, Moges F, Gelaw A, Eshete S, Mekonnen F. Prevalence and antimicrobial susceptibility patterns of extended spectrum beta-lactamase producing Entrobacteriaceae in the University of Gondar Referral Hospital environments, northwest Ethiopia. BMC Res Notes. 2018;11(1):335. doi: 10.1186/s13104-018-3443-1. PubMed PMID: 29788988; PubMed Central PMCID: PMCPMC5964971.

11. Endalafer N, Gebre-Selassie S, Kotiso B. Nosocomial bacterial infections in a tertiary hospital in Ethiopia. Journal of Infection Prevention. 2011;12(1):38-43.

12. Desta K, Woldeamanuel Y, Azazh A, Mohammod H, Desalegn D, Shimelis D, et al. High Gastrointestinal Colonization Rate with Extended-Spectrum $\beta$-Lactamase-Producing Enterobacteriaceae in Hospitalized Patients: Emergence of Carbapenemase-Producing K. pneumoniae in Ethiopia. PloS one. 2016;11(8):e0161685.

13. Yallew WW, Kumie A, Yehuala FM. Point prevalence of hospital-acquired infections in two teaching hospitals of Amhara region in Ethiopia. Drug, healthcare and patient safety. 2016;8:71.

14. Mbanga J, Sibanda A, Rubayah S, Buwerimwe F, Mambodza K. Multi-Drug Resistant (MDR) Bacterial Isolates on Close Contact Surfaces and Health Care Workers in Intensive Care Units of a Tertiary Hospital in Bulawayo, Zimbabwe. Journal of Advances in Medicine and Medical Research. 2018:1-15. 
15. Shiferaw T, Gebr-Silasse L, Mulisa G. Bacterial indoor-air load and its implications for healthcare-acquired infections in a teaching hospital in Ethiopia. International Journal of Infection Control. 2016;12(1).

16. Getachew H, Derbie A, Mekonnen D. Surfaces and air bacteriology of selected wards at a referral hospital, Northwest Ethiopia: a cross-sectional study. International journal of microbiology. 2018;2018.

17. Dancer SJ. How do we assess hospital cleaning? A proposal for microbiological standards for surface hygiene in hospitals. Journal of Hospital Infection. 2004;56(1):10-5.

18. Messai Y, Iabadene H, Benhassine T, Alouache S, Tazir M, Gautier V, et al. Prevalence and characterization of extended-spectrum $\beta$-lactamases in Klebsiella pneumoniae in Algiers hospitals (Algeria). Pathologie Biologie. 2008;56(5):319-25.

19. Chen C-H, Lin Y-L, Chen K-H, Chen W-P, Chen Z-F, Kuo H-Y, et al. Bacterial diversity among four healthcare-associated institutes in Taiwan. Scientific reports. 2017;7(1):8230.

20. Dallolio L, Raggi A, Sanna T, Mazzetti M, Orsi A, Zanni A, et al. Surveillance of environmental and procedural measures of infection control in the operating theatre setting. International journal of environmental research and public health. 2018;15(1):46.

21. Garcia LS. Clinical microbiology procedures handbook: American Society for Microbiology Press; 2010.

22. CLSI. Performance standards for antimicrobial susceptibility testing. 28th ed. Wayne P, editor: Clinical and Laboratory Standards Institute; 2018.

23. Lalami AEO, Touijer H, El-Akhal F, Ettayebi M, Benchemsi N, Maniar S, et al. Microbiological monitoring of environment surfaces in a hospital in Fez city, Morocco Surveillance microbiologique des surfaces de l'environnement d'un hôpital dans la ville de Fès, au Maroc. 2016.

24. Al Laham NA. Prevalence of bacterial contamination in general operating theaters in selected hospitals in the Gaza Strip, Palestine. Journal of infection and public health. 2012;5(1):43-51.

25. Nurain AM, Bilal NE, Ibrahim ME. The frequency and antimicrobial resistance patterns of nosocomial pathogens recovered from cancer patients and hospital environments. Asian Pacific Journal of Tropical Biomedicine. 2015;5(12):1055-9.

26. Sserwadda I, Lukenge M, Mwambi B, Mboowa G, Walusimbi A, Segujja F. Microbial contaminants isolated from items and work surfaces in the post-operative ward at Kawolo general hospital, Uganda. BMC infectious diseases. 2018;18(1):68.

27. Hammuel C, Jatau ED, Whong CM. Prevalence and antibiogram pattern of some nosocomial pathogens isolated from Hospital Environment in Zaria, Nigeria. Aceh International Journal of Science and Technology. 2014;3(3):131-9.

28. Weber DJ, Anderson D, Rutala WA. The role of the surface environment in healthcareassociated infections. Curr Opin Infect Dis. 2013;26(4):338-44. doi: 10.1097/QCO.0b013e3283630f04. PubMed PMID: 23743816.

29. Chemaly RF, Simmons S, Dale Jr C, Ghantoji SS, Rodriguez M, Gubb J, et al. The role of the healthcare environment in the spread of multidrug-resistant organisms: update on current best practices for containment. Therapeutic advances in infectious disease. 2014;2(3-4):79-90.

30. Weber DJ, Rutala WA. Understanding and preventing transmission of healthcareassociated pathogens due to the contaminated hospital environment. Infect Control Hosp Epidemiol. 2013;34(5):449-52. doi: 10.1086/670223. PubMed PMID: 23571359. 
31. Santajit S, Indrawattana N. Mechanisms of Antimicrobial Resistance in ESKAPE Pathogens. Biomed Res Int. 2016;2016:2475067. doi: 10.1155/2016/2475067. PubMed PMID: 27274985; PubMed Central PMCID: PMCPMC4871955.

32. Alemu A, Misganaw D, Wondimeneh Y. Bacterial profile and their antimicrobial susceptibility patterns of computer keyboards and mice at Gondar University Hospital, Northwest Ethiopia. Biomed Biotechnol. 2015;3(1):1-7.

33. Tajeddin E, Rashidan M, Razaghi M, Javadi SS, Sherafat SJ, Alebouyeh M, et al. The role of the intensive care unit environment and health-care workers in the transmission of bacteria associated with hospital acquired infections. J Infect Public Health. 2016;9(1):13-23. doi: 10.1016/j.jiph.2015.05.010. PubMed PMID: 26117707.

34. Maryam A, Hadiza U-S, Aminu UM. Characterization and determination of antibiotic susceptibility pattern of bacteria isolated from some fomites in a teaching hospital in northern Nigeria. African Journal of Microbiology Research. 2014;8(8):814-8.

35. Rohr U, Kaminski A, Wilhelm M, Jurzik L, Gatermann S, Muhr G. Colonization of patients and contamination of the patients' environment by MRSA under conditions of singleroom isolation. Int J Hyg Environ Health. 2009;212(2):209-15. Epub 2008/08/01. doi: 10.1016/j.ijheh.2008.05.003. PubMed PMID: 18667356.

36. Ekrami A, Kayedani A, Jahangir M, Kalantar E, Jalali M. Isolation of common aerobic bacterial pathogens from the environment of seven hospitals, Ahvaz, Iran. 2011.

37. Faires MC, Pearl DL, Berke O, Reid-Smith RJ, Weese JS. The identification and epidemiology of meticillin-resistant Staphylococcus aureus and Clostridium difficile in patient rooms and the ward environment. BMC Infect Dis. 2013;13(1):342. doi: 10.1186/1471-2334-13342. PubMed PMID: 23883171; PubMed Central PMCID: PMCPMC3727943.

38. Faires MC, Pearl DL, Ciccotelli WA, Straus K, Zinken G, Berke O, et al. A prospective study to examine the epidemiology of methicillin-resistant Staphylococcus aureus and Clostridium difficile contamination in the general environment of three community hospitals in southern Ontario, Canada. BMC Infect Dis. 2012;12(1):290. doi: 10.1186/1471-2334-12-290. PubMed PMID: 23136936; PubMed Central PMCID: PMCPMC3532087.

39. Mengistu H, Misganaw B, Elshaday A. Bacterial load and antibiotic susceptibility pattern of isolates in operating rooms at Hawassa University Referral Hospital, southern Ethiopia. Journal of Microbiology and Antimicrobials. 2016;8(1):1-6. doi: 10.5897/jma2015.0349.

40. Tong SY, Davis JS, Eichenberger E, Holland TL, Fowler VG. Staphylococcus aureus infections: epidemiology, pathophysiology, clinical manifestations, and management. Clinical microbiology reviews. 2015;28(3):603-61.

41. Fagade OE, Ezeamagu CO, Oyelade AA, Ogunjobi AA. Comparative study of antibiotic resistance of Staphylococcus species isolated from clinical and environmental samples. Assumption University J Tech. 2010;13(3):165-9.

42. Salm F, Deja M, Gastmeier P, Kola A, Hansen S, Behnke M, et al. Prolonged outbreak of clonal MDR Pseudomonas aeruginosa on an intensive care unit: contaminated sinks and contamination of ultra-filtrate bags as possible route of transmission? Antimicrobial Resistance \& Infection Control. 2016;5(1):53.

43. Parcell BJ, Oravcova K, Pinheiro M, Holden MTG, Phillips G, Turton JF, et al. Pseudomonas aeruginosa intensive care unit outbreak: winnowing of transmissions with molecular and genomic typing. J Hosp Infect. 2018;98(3):282-8. doi: 10.1016/j.jhin.2017.12.005. PubMed PMID: 29229490; PubMed Central PMCID: PMCPMC5840502. 
534 44. Kramer A, Schwebke I, Kampf G. How long do nosocomial pathogens persist on 535 inanimate surfaces? A systematic review. BMC infectious diseases. 2006;6(1):130.

536 45. Ensayef S, Al Shalchi S, Sabbar M. Microbial contamination in the operating theatre: a 537 study in a hospital in Baghdad. 2009.

538 46. Sui Y-S, Wan G-H, Chen Y-W, Ku H-L, Li L-P, Liu C-H, et al. Effectiveness of bacterial 539 disinfectants on surfaces of mechanical ventilator systems. Respiratory care. 2012;57(2):250-6.

540 47. $\mathrm{Hu} \mathrm{L}$, Liu Y, Deng L, Zhong Q, Hang Y, Wang Z, et al. Outbreak by ventilator541 associated ST11 K. pneumoniae with co-production of CTX-M-24 and KPC-2 in a SICU of a 542 tertiary teaching hospital in central China. Frontiers in microbiology. 2016;7:1190.

543 48. Martin RM, Bachman MA. Colonization, infection, and the accessory genome of 544 Klebsiella pneumoniae. Frontiers in cellular and infection microbiology. 2018;8:4.

545 49. Davies J, Davies D. Origins and evolution of antibiotic resistance. Microbiol Mol Biol 546 Rev. 2010;74(3):417-33. doi: 10.1128/MMBR.00016-10. PubMed PMID: 20805405; PubMed 547 Central PMCID: PMCPMC2937522.

548 50. Eshetie S, Tarekegn F, Moges F, Amsalu A, Birhan W, Huruy K. Methicillin resistant 549 Staphylococcus aureus in Ethiopia: a meta-analysis. BMC Infect Dis. 2016;16(1):689. doi: 550 10.1186/s12879-016-2014-0. PubMed PMID: 27871245; PubMed Central PMCID: 551 PMCPMC5117566.

552 51. Sievert DM, Ricks P, Edwards JR, Schneider A, Patel J, Srinivasan A, et al. 553 Antimicrobial-resistant pathogens associated with healthcare-associated infections summary of 554 data reported to the National Healthcare Safety Network at the Centers for Disease Control and 555 Prevention, 2009-2010. Infection Control \& Hospital Epidemiology. 2013;34(1):1-14.

556 52. Loomba PS, Taneja J, Mishra B. Methicillin and vancomycin resistant S. aureus in 557 hospitalized patients. Journal of global infectious diseases. 2010;2(3):275. 